\title{
ERRATUM
}

\section{Brief Report: Coronary Heart Disease Events Associated with Hormone Therapy in Younger and Older Women} A Meta-Analysis

\author{
Shelley R. Salpeter, $M D^{1,2,3}$, Judith M. E. Walsh, MD, $\mathrm{MPH}^{4}$, Elizabeth Greyber, $\mathrm{MD}^{7}$, \\ and Edwin E. Salpeter, PhD ${ }^{5}$ \\ 'Department of Medicine, Santa Clara Valley Medical Center, San Jose, CA, USA; ${ }^{2}$ Stanford University School of Medicine, Stanford, CA, USA; \\ ${ }^{3}$ Department of Medicine, Santa Clara Valley Medical Center, San Jose, CA, USA; ${ }^{4}$ Department of Medicine, University of California, San \\ Francisco, San Francisco, CA, USA; ${ }^{5}$ Department of Space Sciences, Cornell University, Ithaca, NY, USA.
}

$\mathrm{J}$ Gen Intern Med 23(10): 1728

DOI: $10.1007 / \mathrm{s} 11606-008-0762-2$

(c) Society of General Internal Medicine 2008

In the article by Salpeter et al. published in the April 2006 issue of the Journal of General Internal Medicine (volume 21, issue 4), the conflict of interest statement published in the article stated, "The institutions had no role in the design, conduct, or reporting of the study. No sponsorship from industry was provided to conduct this analysis." However, the manuscript as accepted by the Editors contained the following conflict of interest statement, "Dr. Salpeter served as a consultant for Wyeth Pharmaceuticals, and was paid on an hourly basis. Wyeth had no role in the design, conduct or reporting of the study, and did not have access to the data." It is unclear where in the editorial process the original conflict of interest statement was changed, but the second conflict of interest statement is the appropriate one and should have been published in the original article. We regret the error.

- The Editors

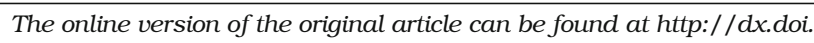
org/10.1111/j.1525-1497.2006.00389.x

Published online August 27, 2008 